\title{
The GAs Extraction and Analyses system (GAEA) for immediate extraction and measurements of volatiles in the Hayabusa2 sample container
}

Yayoi N. Miura ( $\square$ yayoi@eri.u-tokyo.ac.jp )

University of Tokyo: Tokyo Daigaku https://orcid.org/0000-0003-0945-2444

Ryuji Okazaki

Kyushu University - Ito Campus: Kyushu Daigaku

Yoshinori Takano Japan Agency for Marine-Earth Science and Technology: Kaiyo Kenkyu Kaihatsu Kiko

Kanako Sakamoto

Japan Aerospace Exploration Agency

Shogo Tachibana

University of Tokyo: Tokyo Daigaku

Keita Yamada

Tokyo Institute of Technology: Tokyo Kogyo Daigaku

Saburo Sakai

Japan Agency for Marine-Earth Science and Technology: Kaiyo Kenkyu Kaihatsu Kiko

Hirotaka Sawada

Japan Aerospace Exploration Agency

\section{Research Article}

Keywords: Hayabusa2, sample return, C-type asteroid, volatiles, gas analysis

Posted Date: January 31st, 2022

DOI: https://doi.org/10.21203/rs.3.rs-1257103/v1

License: (c) (i) This work is licensed under a Creative Commons Attribution 4.0 International License.

Read Full License 


\section{Abstract}

Hayabusa2 returned surface samples from the C-type near-Earth asteroid (162173) Ryugu to Woomera, South Australia, in December 2020. The samples returned from Ryugu are expected to contain volatile components reflecting its primitive nature and solar wind components due to exposure to space. Such volatiles may partly be released inside the sealed sample container enclosing Ryugu samples due to particle destruction or container heating in a contingency case. In order to collect and analyze volatiles released in the container prior to the container opening, we set up a gas extraction and analyses system (GAEA: GAs Extraction and Analyses system). The system requires ultra-high vacuum condition, small vacuum line volume to minimize dead volume and simple configuration as well as having an interface to connect the container. The system includes gas bottles for passive collection of volatiles at room temperature and bottles for active collection at liquid nitrogen temperature. A quadrupole mass spectrometer is installed to analyze gases in the vacuum line, and a non-evaporative getter pump is also used when noble gases are analyzed. The rehearsal operation of GAEA was made at ISAS/JAXA as well as transportation tests in Japan. In November 2020, it was transported safely to the Quick Look Facility (QLF) in Woomera. It was set up at the QLF and worked well as planned for collection and analysis of gas components from the returned sample container. Here we report the concept, design and calibration results for the GAEA and an outline of analytical protocols applied in Woomera.

\section{Introduction}

Hayabusa2 is the second asteroidal sample return mission operated by Japan Aerospace Exploration Agency (JAXA). It launched on December 3, 2014 and arrived at the target asteroid (162173) Ryugu on June 27, 2018. Ryugu is a C-type near-Earth asteroid covered with regolith that also involves pebbles and sands (Tachibana et al., 2021 a and references therein). C-type asteroids have been considered to record the early evolution of the Solar System in their primitive constituents including volatiles (Tachibana et al., 2014; Watanabe et al., 2017). Science objectives of the Hayabusa2 mission are to investigate (1) evolution from a planetesimal to a near-Earth asteroid (i.e., thermal processes in a planetesimal in the early solar system, heating and space weathering on the surface of near-Earth asteroid at its current orbit), (2) potential destruction and accumulation of a rubble-pile body that formed from a larger aqueously altered parent planetesimal (i.e., planetesimal formation and impact processes throughout the solar system history), (3) diversification of organic materials through interactions with minerals and water in a planetesimal (i.e., origin and evolution of volatile components in the early solar system and final state of organic matter and water prior to their delivery to the rocky planets) and (4) chemical heterogeneity in the early solar system (i.e., mixing of high-temperature and low-temperature components during dynamical evolution of the proto-solar disk) (e.g., Tachibana et al., 2014; Watanabe et al., 2017). In order to clarify these objectives, the volatile components are very important targets to be analyzed on ground. It has been known that primitive meteorites contain water and a variety of organic matters synthesized from volatile elements such as $\mathrm{H}, \mathrm{C}, \mathrm{N}$ and $\mathrm{O}$. Such water and organics could have been related to a possible source of Earth's ocean and life. In addition, implanted solar wind and galactic 
cosmic-ray produced nuclides are likely existed as like returned samples from the asteroid Itokawa (Nagao et al., 2011; Busemann et al., 2015) and lunar samples (Wieler, 2016 and references therein), from which surface histories such as gardening efficiency and time scale of movement of surface material would be constrained. Small fractions of solar wind noble gases were released at low temperatures like $100{ }^{\circ} \mathrm{C}$ for lunar samples and gas-rich meteorites (e.g., Black, 1972a, 1972b; Pepin et al., 1999), therefore, not only components kept in solid samples but also those released into gas phases in the sample container should be recovered and analyzed.

In order to reduce terrestrial contamination, we planned that the volatile gases in the sample container are collected in gas bottles and measured with a quadrupole mass spectrometer (QMS) soon after the recovery of the Sample Return Capsule (SRC) in Australia. The Hayabusa2 sample container has a metalto-metal sealing system and a gas sampling interface to extract volatiles without exposure to the terrestrial atmosphere, which were developed based on the lessons learned from the Hayabusa mission (Okazaki et al., 2017; Sawada et al., 2017). Another motivation of gas extraction from the sample container before opening is to keep the interior pressure as low as possible to avoid the loss of sample due to gas stream induced by pressure difference between the container and the vacuum clean chamber dedicated to Hayabusa2 samples at ISAS/JAXA. This motivation also came from the lessons learned from the Hayabusa mission, where the poorly constrained container pressure prevented prompt container opening in a clean chamber (Okazaki et al., 2011).

Hayabusa2 carried out remote sensing observations on Ryugu with findings of $\mathrm{OH}$-related absorption and touchdown operations at two landing sites for sample collection (e.g., Sugita et al., 2019; Arakawa et al., 2020; Morota et al., 2020; Tsuda et al., 2020; Kameda et al., 2021; Kitazato et al., 2021; Okada et al., 2021; Tachibana et al., 2021a; Tatsumi et al., 2021). Then, Hayabusa2 left Ryugu on November 13, 2019 (Saiki et al., 2021). After its journey towards Earth, the spacecraft successfully delivered the SRC, which landed at Woomera desert in Australia on December 6, 2020 (Yamada et al., 2021). The SRC was found 2 hours after its landing and immediately carried to the Quick Look Facility (QLF) in Woomera Prohibited Area (WPA), South Australia (Tachibana et al., 2021b). The sample container, in which the collected samples were stored, was taken from the SRC and set on a baseplate of the ICF356 flange (Fig. 1). Then, it was connected to the GAs Extraction and Analyses system (GAEA) via an ICF34 flange of the metal valve in order to extract, collect and measure volatile components in the sample container before the containeropening at ISAS/JAXA in Sagamihara, Japan. In this paper, (i) the concept and configuration of the GAEA and (ii) an outline of the analytical procedure at the QLF in Woomera are described.

\section{Gas Extraction And Analyses Sytem (Gaea)}

\section{System concept and requirements}

The entire system was designed based on the concepts of (a) keeping an ultra-high vacuum condition using oil free pumps, (b) small line volumes to minimize dead volume, (c) introducing a programable valve control system to avoid human errors and against accidental power failure, and (d) simple, firm and 
easy-handling configuration to be transported between Japan and Australia. The system was required to fit within $1.5 \mathrm{~m} \times 1.5 \mathrm{~m} \times 1.5 \mathrm{~m}$ in size and to be operated with $10 \mathrm{kVA}$. As basic performance, dynamic (non-static) pressures of analytical lines were requested to be $<1 \times 10^{-5}$ and $<1 \times 10^{-8} \mathrm{~Pa}$ at a gas extraction line and near turbo molecular pumps, respectively, within 150 hours after re-starting pumps. These are important to execute certainly and promptly the volatile analysis for Hayabusa2 container gases in Australia within limited time, space, and power supply. In order to configure the system that satisfies the requirements, we performed some preliminary experiments and then designed the system with careful consideration.

\section{Development and preliminary experiments}

We developed an original gas extraction method, that is gases are extracted from the sample container without exposure of the terrestrial atmosphere by making a hole at the bottom of the container (HAWKS; HAyabusa2 gas collection With the Keyhole System). The principal of the HAWKS was reported in Okazaki et al. (2017). Schematic draws of the gas sampling interface of the sample container and the final design of the needle are shown in Fig. 2. Our advance experiments acquired before the launch of the Hayabusa2 indicated that a hole can be made without any leakage in the sealing between the container and the base flange and any mechanical troubles such as stacking. For more certain execution to be performed in Woomera, we reexamined suitable shape of the tungsten carbide needle and feed stroke of the needle. Because the clearance between the bottom plate of the container and a filter set above the plate is $\sim 0.85 \mathrm{~mm}$ (Fig. 2c), the moving distance by the liner feedthrough must be controlled with an accuracy far better than $\pm 1 \mathrm{~mm}$ not to break the filter. Ten designs of needles with different sizes were prepared and tested: two types of the corn head angle $\left(30\right.$ and $\left.45^{\circ}\right)$, three types of corn head sharpness (sharp, 0.3 and $0.5 \mathrm{~mm}$ in diameter), four sizes of cylindrical shape heads at the top of the corn heads $(0.3,0.5,0.6$ and $0.7 \mathrm{~mm}$ in diameter) and some combinations of them. Tested results showed slightly but significantly different hole morphologies. Among them, a needle making a whole most flatly and smoothly was selected for the actual operation (Fig. 2d). Its body diameter is $3 \mathrm{~mm}$, and the corn head angle is $30^{\circ}$. The tip of needle has a delta corn shape with a pin-like protuberance $(0.6 \mathrm{~mm}$ in height and $0.6 \mathrm{~mm}$ in diameter). The stroke of moving the needle, by measuring the micro scale of the feedthrough, was determined to be $1.2 \mathrm{~mm}$ through preliminary tests using simulated sample containers. The gases in the sample container were successfully extracted using the HAWKS in Woomera.

The Hayabusa2 sample container has approximately $200 \mathrm{~cm}^{3}$ in volume. To collect most of the gases stored in the sample container (e.g., $~ 90 \%$ of the gases) in a reasonable size of four bottles, the line volume of gas-expanding-areas is likely to be $\leq 100 \mathrm{~cm}^{3}$. For this, we considered to use pipes with small inner diameter. However, because pipes with small inner diameter have smaller conductance, pumping efficiency was a matter of concern. Therefore, we examined pumping efficiency and ultimate vacuum pressure using pipes with two different inner diameters of $\sim 11$ and $\sim 6 \mathrm{~mm}$. The two test pipes having a few ten $\mathrm{cm}$ long with three corners, each corner must hinder the pumping speed, showed an order of magnitude different vacuum pressures at the gauge sited in the vacuum lines. We also tested if there is a pressure difference by the effect of hydrogen permeation using thin diameter pipes having different pipe 
thicknesses, $\sim 1.7 \mathrm{~mm}$ and $14 \mathrm{~mm}$ in pipe thickness, respectively. The results of the vacuum pressures at the end of each pipe are given in Fig. 3, showing no notable difference. After bake-out at $200{ }^{\circ} \mathrm{C}$ several times, the pressures observed at the end of these pipes reached $\leq 5^{\prime} 10^{-6} \mathrm{~Pa}$ (Fig. 3c). The hydrogen permeation into the line throughout the pipe wall was not effective even for the small-bore pipe with the thin thickness. Considering these observations, we designed the GAEA using thin pipe ( $6.5 \mathrm{~mm}$ in inner diameter) with thin thickness $(\sim 1.2 \mathrm{~mm})$ for main pipes with straight line structure and short length as much as possible.

For pressure monitor, cold cathode gauges (CCG) are used near the turbo molecular pumps because of their quick response and easy usage for monitoring wide range pressures. On the other hand, a miniature ion gauge (MG) is chosen at a gas-expanding-area to measure pressures of gases released from the sample container. The CCGs have relatively low accuracy (accuracy decreases with operation time) due to cathode sputtering and plasma-chemical reactions that causes contamination within the gauge (Wilfert, 2003) and no function to avoid such contamination. Moreover, the CCGs evacuate ionized gases, and its evacuation efficiency was found not to be negligibly small. Therefore, a miniature ion gauge is adopted for monitoring middle and high vacuum ranges after the first monitor with a pirani gauge (PG).

A quadrupole mass spectrometer (QMS) is used for identification of volatile constituents. QMS has advantages of easy operation and quick measurements. We also preliminary examined background mass spectra for three commercial QMS, among which one (Tokyo Electronics, Watmass MPH200M) that presented the least abundant background spectra was selected. In addition, we introduced another QMS to use leak check after setting the sample container.

As mentioned, one possible important target to be analyzed is solar wind noble gases, particularly He and $\mathrm{Ne}$. In order to remove active gases, a SORB-AC getter (non-evaporative getter pump; hereafter SORB-AC) is set. It can be used before or during QMS measurements by opening a valve. It is also helpful to evaluate contribution of active gases, such as hydrocarbon, nitrogen and oxide molecules, by comparing mass spectra with and without SORB-AC. Essentially, only noble gases are not trapped by SORB-AC, which means peak intensities of noble gases are similar between with and without SORB-AC.

\section{Instrument descriptions}

A schematic figure and an external view of the GAEA are shown in Figs. 4 and 5, respectively. The system is composed of

- metal lines (mostly made of SUS 316) and metal valves (VACGEN ZCR20 and Swagelok SS-4H) for ultra-high-vacuum

- a connecting port to attach the Hayabusa2 sample container that is mounted on an ICF356 flange with an ICF34 port (Fig. 1)

- pressure gauges to monitor extracted gases and vacuum conditions of the system (Ulvac GP1000G, Anelva MG-2 and Pfeiffer IKR27) 
- a SORB-AC getter (SAES NP-10)

- six bottles for storing the container gases at room temperature (bottles at normal temperature, a design draw is given in Additional file 1: Fig. S1a) and three bottles for trapping the gases at the liquid nitrogen temperature (bottles for cold-trap, Fig. S1b), among them each one bottle is used to collect background gases of the GAEA at room temperature and at the liquid nitrogen temperature.

- two quadrupole mass spectrometers (QMS): (i) QMS (W); main QMS for container gas analyses (Tokyo Electronics, Watmass MPH 200M, for which Inficon Transpector MPH200M is used as the base model and the operation software is Inficon FebGuard Explorer), and (ii) QMS (A) used for leak check (Anelva M-201QA-TDM)

- three vacuum pump sets, each of which consists of a turbo molecular pump (Shimazu TMP-B300), a dry scroll pump (Edwards nXDS10i), a solenoid operation isolation valve (Edwards PV25EK) and a pirani gauge (Ulvac PG1000G)

- a power supply controller

- motor drive devices for metal valve operation (ZCR20 operatable automatically using a motor drive or on manual, and SS-4H for manual operation)

- heaters and controllers to bake-out the vacuum lines

The main power voltage for the system is $100 \mathrm{~V}$ except for baking heaters and motor drives to operate the ZCR20 values. The power supply distribution is schematically drawn in Fig. 6. Transformers to step down voltage from $240 \mathrm{~V}$ to $100 \mathrm{~V}$ are prepared for operation in Australia. Three UPSs (uninterruptible power supplies; GS Uasa THA5000-10, Omuron BU200RW) are also provided as backup power sources for any accidental power cut. For system safety, all the ZCR20 valves are automatically closed with alerts for power cut (a UPS starts) or unusual decrease of a vacuum pressure.

The line volumes used for gas expansion, colored in blue on Fig. 4, were determined by a method based on Boyle's law; gas pressures were measured by a manometer for individual volume areas alone and combined with a reference part whose absolute volume was determined by weighing filled water beforehand. The volumes of each line area are summarized in Table 1. The gas-storage bottles at normal temperature (NT \#1 - \#6) and those for liquid nitrogen temperature (CR \#1 - \#3) have the volumes of 750 and $50 \mathrm{~cm}^{3}$, respectively (Additional file 1: Fig. $\mathrm{S} 1$ for their design draws). The Hayabusa2 sample container and a flexible tube connecting between the container and the GAEA have approximate volumes of 200 and $15 \mathrm{~cm}^{3}$, respectively. Volume factors of gas reduction and fractions of gases collected in the gas-storage bottles are calculated using the line volumes and summarized in Table 2, based on which we can evaluate the original gas amount inside the sample container. Among the extracted gases from the container, $80 \%$ is stored in four bottles of NT \#1 - \#4 (20\% in each bottle). Remaining gases are used for the measurements with QMS (W), the collection in two bottles of CR \#1 - \#2 as cold-trap gases at the liquid nitrogen temperature and re-analyses (stored in NT \#6 and re-measured with QMS (W)).

\section{Pre-operation and calibration at ISAS}


The GAEA was installed at the Sagamihara campus of ISAS/JAXA in 2019. Photographs of the GAEA set at the ISAS/JAXA (Sagamihara, Japan) and the QLF (Woomera, Australia) are shown in Fig. 7 and Additional file 1: Fig. S2. After confirmation of the initial condition, the system was moved to Kyushu University for improvements and a transportation test on November 28, 2019. The system was transported safely back to ISAS/JAXA on June 22, 2020 and was set up again for the operation test and training. From June to September 2020, bake-out of the system, calibration and some adjustments for stable operation were carried out. The regular vacuum pressures reached down to $1 \times 10^{-8}, 5 \times 10^{-9}$ and 7 $\times 10^{-9} \mathrm{~Pa}$ for CCG1, CCG2 and CCG3, respectively (see Fig. 4 for the system layout).

The sensitivity of the QMS (W) was determined for ${ }^{40} \mathrm{Ar}$ by introducing air into the system. Air is a mixture of $78 \mathrm{vol} \%$ of $\mathrm{N}_{2}, 21 \mathrm{vol} \%$ of $\mathrm{O}_{2}$ and $0.93 \mathrm{vol} \%$ of $\mathrm{Ar}$, water vapor and some other gases as minor components. Argon has three stable isotopes ${ }^{36} \mathrm{Ar},{ }^{38} \mathrm{Ar}$ and ${ }^{40} \mathrm{Ar}$ (molar fractions of each isotope are $0.0034,0.00063$ and 0.996 , respectively). The amount of air introduced in the system was required to be minimal to keep the background level as low as possible. For input of the gases into the system, a small tank containing the quantitative amount of air was attached at the ICF34 flange next to the line named [VGauge], to which the Hayabusa2 sample container mounted on the ICF356-baseplate is connected via [VFL] (the flange port and [V-FL] had never been used before the actual operation in Woomera). The QMS (W) has two detector modes, the faraday-cup mode (lower sensitivity) and the SEM mode (higher sensitivity). The SEM mode was used for measurements in nominal operation. The operating condition of the QMS (W) was $2 \mathrm{~mA}$ for the emission current, $70 \mathrm{eV}$ for the electron energy and $900 \mathrm{~V}$ for the secondary electron multiplier voltage of the detector, respectively. The calibration data are summarized in Table 3. The amounts of the atmosphere introduced into the QMS (W) were $1.7 \times 10^{-8}-2.8 \times 10^{-7}$ $\mathrm{cm}^{3}$ STP $\left(1.6 \times 10^{-10}-2.6 \times 10^{-9} \mathrm{~cm}^{3}\right.$ STP for $\left.{ }^{40} \mathrm{Ar}\right)$. The pressures in the QMS (W) during measurements were calculated to be $3.9 \times 10^{-6}-6.6 \times 10^{-5} \mathrm{~Pa}$ based on the amounts of the gases and the expanding volumes. Representative mass spectra for the calibration (run numbers of 10(c), 11(a) and 12) and a blank run before the calibration 11(a) are shown in Fig. 8. In the calibration measurements, peak scans of $\mathrm{m} / \mathrm{z}$ from 1 to 100 were conducted. The peak scan was started when gas was introduced into the QMS (W). The peak intensities were stabilized after the third scan, around $55 \mathrm{sec}$ after the gas introduction, and then decreased in general. The mass spectra were first obtained without SORB-AC, and after 2 min those were obtained with SORB-AC by opening the valve at SORB-AC (Fig. 4). The peak at $m / z=40$ did not change significantly between with and without SORB-AC, implying the peaks to be entirely ${ }^{40} \mathrm{Ar}$. The amounts of ${ }^{40} \mathrm{Ar}$ in blank measurements were comparable to the detection limit $\left(\sim 7 \times 10^{-12} \mathrm{~cm}^{3}\right.$ STP $)$ during the period of these calibration measurements. The mass spectra of the calibration and blank measurements show that there were no significant peaks higher than $m / z=47$. For calculating the sensitivities, (i) the intensities of the third scan (around $55 \mathrm{sec}$ after the air introduction) were used, (ii) blank corrections were applied, and (iii) sensitivities were unified as the same volume condition of [V-PQ + V-QMS + QMS (W)] (Fig. 4) during the QMS measurements by volume corrections. The obtained sensitivities are listed in Table 3. The average of the sensitivities of ${ }^{40} \mathrm{Ar}$ was determined to be $650 \pm 120$ $\mathrm{cm}^{3}$ STP/A by twelve measurements from July 8 to September 16,2020 . The intensities of $\mathrm{m} / \mathrm{z}=28$ (I28) 
relative to $m / z=40$ (140) are also shown in Table 3. The peaks of $m / z=28$ are likely a mixture of $\mathrm{N}_{2}$ and CO. Although the ratios of I28/140 were variable, calibration runs on July 21 and August 18, 2020 at which the blank levels were relatively low, provided consistent I28/140 ratios with the average of $94 \pm 6$. This ratio is close to the $\mathrm{N}_{2} /{ }^{40} \mathrm{Ar}$ ratio of the terrestrial atmosphere $\left(\mathrm{N}_{2} /{ }^{40} \mathrm{Ar}=84 ;\right.$ Ozima and Podosek, 2002), suggesting that the relative sensitivity between $\mathrm{N}_{2}$ and ${ }^{40} \mathrm{Ar}$ is close to 1 for the QMS (W). The mass spectra indicate that the species originated from calibration air were not significant other than $\mathrm{Ar}$ and $\mathrm{N}_{2}$ beyond blank levels. Though $\mathrm{O}_{2}$ is the second abundant gases (the mole fraction is 0.209 , that is $\sim 22$ times of $\mathrm{Ar})$, the signal of $\mathrm{O}_{2}(\mathrm{~m} / \mathrm{z}=32)$ was almost similar to that for the blanks, which were 1-10 times of Ar. The oxygen molecules were possibly dissociated by filaments of the QMS (W) or the ion pressure gauge, or alternatively, adsorbed on wall surfaces inside of the stainless-steel pipes via physic-chemical reaction such as oxidation. Considering the intensities of $m / z=32$ being stable soon after gas introduction, oxygen could have decreased before input into the QMS (W), however, it is unclear what was the mechanism. The empirical sensitivities relative to Ar were calculated to be 1.3, 1.5, 1.2 and 1.0 for $\mathrm{N}_{2}$, $\mathrm{CO}, \mathrm{O}_{2}$ and $\mathrm{CO}_{2}$, respectively, according to previous studies on ionization probabilities, fragmentation factors and transmitting efficiencies of a quadrupole mass filter (cf., Summers, 1969; Lieszkovsky et al., 1990; Jousten, 2008; Malyshev and Middleman, 2008; Yoshida and Arai, 2018; Operating manual of Transpector MPH gas analysis system, PN 074-555-P1C, Inficon). The relative sensitivities reported for QMS in previous studies indicate more or less variation, but those are within $1 \pm \sim 50 \%$ for the above species in most cases (e.g., Mahaffy et al., 2011; Hirashita et al., 2013; Yoshida et al., 2014; Sun et al., 2020). Uncertainties arisen from long term stabilization of sensitivities, pressure dependence, cracking parameters of molecules during mass spectrometry and so on might be larger than deviations of the relative sensitivities from the unity. We here assumed the relative sensitivity factors for all gaseous species are 1 with the $50 \%$ uncertainty.

\section{The Analytical Procedure At The QIf In Woomera}

An outline of the analytical protocols for extraction, storage and measurements of volatiles gases from the Hayabusa2 sample container is shown in Fig. 9. After the recovery and safety check operation of the SRC, the sample container is taken out from it at the QLF in WPA. The outside of the container is wiped with acetone and ethanol. The container is mounted on the ICF356 flange via a metal O-ring (Usui Co. Ltd., U-tightseal CS10033AI00G50, whose outer diameter, inner diameter, wire diameter and groove depth are $\varphi 57.6, \varphi 51.0, \varphi 3.3$ and $0.5 \mathrm{~mm}$, respectively). Then, it is connected to the GAEA through the flexible tube named [V-FL] (Fig. 1). The atmosphere in the area between the sample container and the GAEA is first evacuated. Overnight evacuation and bake-out of the vacuum line of the GAEA are conducted, during which the sample container is not yet evacuated and heated. When the vacuum pressure of the line becomes low enough for analyses $\left(\leq 10^{-7} \mathrm{~Pa}\right)$ and no leak is detected at the connection flanges by helium leak check, gases are extracted through a hole made at the bottom of the container by moving a tungsten-carbide needle attached on a linear motion feed-through (Fig. 1). The extracted gases are expanded in the volume of [V-Container + V-FL + V-Gauge] with pressure monitoring with PG4. Then, the 
gases are expanded up to [V-PQ], and the gases in [V-PQ] are kept for measurements with the QMS (W). The fraction of [V-PQ] is to be $4.3 \%$ of the gases released from the container. The gases in the other volumes are expanded to gas bottle areas of [V-NTTP + four gas bottles NT \#1 - \#4]. The two valves for each bottle are closed to store the gases at room temperature. The four bottles should thus contain gases with the same amounts and compositions. The fraction stored in the four bottles is to be $80 \%$ of the extracted gases, meaning that each bottle contains $20 \%$ of the gases released from the container. The gases in [V-Container + V-FL] are held by closing the valve between [V-FL] and [V-Gauge], which are used for re-analysis later. The residual gases in the line are expanded and sequentially trapped into two gas bottles, CR \#1 and \#2, cooled with liquid nitrogen at a $50 \%$-height level from the bottom of the bottles for 2 min and $80 \%$-height level for another 2 min. During gas trapping at CR \#1 and \#2, the pressure changes are monitored with PG4. Then, the gases stored in [V-PQ] are expanded to [V-Gauge + V-1 + V-2 + $\mathrm{V}-\mathrm{PQ}]([\mathrm{V}-\mathrm{Gauge}+\mathrm{V}-1+\mathrm{V} 2]$ is evacuated in advance), and reduction procedures coupling the line areas (e.g., [V-PQ]/ [V-Gauge + V-1 + V-2 + V-PQ]) are applied before the QMS measurements when the pressure of the gases are higher than the maximum operatable pressure for the QMS $(W)\left(\sim 10^{-2} \mathrm{~Pa}\right)$. The fractions of the gases collected in the bottles and in [V-PQ] are shown in Table 2.

For the measurements with the QMS $(\mathrm{W})$, mass spectra are obtained with the peak scan mode $(\mathrm{m} / \mathrm{z}$ from 1 to 100 for nominal operation, and from 1 to 200 as an option) and the peak jumping mode for characteristic gases such as hydrogen, helium, carbon, methane, and water depending on gases detected with the peak scan mode. The procedure files for these measurements (recipes $1-5$ ) are set in the operation software of the QMS (W) (Table 4). When gases are introduced into the QMS (W), the valve at the SORB-AC getter is kept in the close state first. The gases are firstly analyzed using the recipes 1 and 2 without SORB-AC and then using the recipes 3 and 4 with SORB-AC by opening the valve at SORB-AC.

Peak scans of $m / z$ from 1 to 200 , the recipe 5 , are performed when various intense peaks are observed at $\mathrm{m} / z \leq 100$ and molecules with $\mathrm{m} / z>100$ are possibly expected to exist. From the obtained data that are given as intensities in the unit of ampere (A), the gas amounts are calculated using the sensitivity of $650 \pm 120 \mathrm{~cm}^{3}$ STP/A for ${ }^{40} \mathrm{Ar}$ and the relative sensitivity factor to ${ }^{40} \mathrm{Ar}$ of 1 as mentioned above. Because the intensities change with time during measurements in the QMS, the absolute amounts should be determined from stabilized data after gas input into the QMS, likely scan data after 54 - 120 sec from the beginning of measurement with the recipe 1 . On the other hand, although the absolute intensities often change with time depending on the gas pressure and the vacuum condition in a mass spectrometer, intensities relative to another such as ${ }^{40} \mathrm{Ar}$ do not change largely unless any condition-change like opening the valve of SORB-AC. Therefore, relative intensities are calculated by averaging the data measured with peak jumping modes that have longer integration time; the results obtained by the recipe 2 (without SORB-AC) are used except for Ar isotope ratios, and data by the recipe 4 (with SORB-AC) are more reliable for Ar because possible interferences by hydrocarbons are reduced.

The SRC-recovery was successfully performed by the Hayabusa2 capsule recovery team. After that, the extraction and measurements of volatile components in the sample container taken from the SRC were also completed at the QLF in Woomera as we planned. In this study, we have established a methodology 
using the GAEA, which is a promising prospect in combination with the successful recovery of Ryugu samples (Tachibana et al., 2021a) and non-destructive analysis of solid samples (Yada et al., 2021; Pilorget et al., 2021). Results on the volatile compositions measured with the QMS and those in the gas bottles, collected at room temperature and with liquid nitrogen, will be reported elsewhere (e.g., Okazaki et al., submitted to Science).

\section{Summary}

Hayabusa2 collected surface materials from the C-type asteroid Ryugu to understand origin and evolution of the asteroid and the Solar System as well as to investigate primitive solar system materials. The collected samples were stored in the sample container, which was sealed by the metal-to-metal sealing system. In order to extract and measure volatile gases in the sample container without exposure to the atmosphere, we designed and constructed the system of ultra-high vacuum line equipping the interface with the sample container, the gas-storage bottles and the QMS (GAEA; The GAs Extraction and Analyses system). The system was set at ISAS/JAXA for pre-operation and training. After bake-out of the system and calibration measurements using the quantitative terrestrial atmosphere, (i) nominal vacuum pressures were $\leqslant 1^{\prime} 10^{-8} \mathrm{~Pa}$ and $\leqslant 1^{\prime} 10^{-6} \mathrm{~Pa}$ at the evacuation lines and gas extraction area, respectively, and (ii) the sensitivity of ${ }^{40} \mathrm{Ar}$ was obtained to be $650 \pm 120 \mathrm{~cm}^{3}$ STP/A. Then, it was transported to Australia for the actual operation. The Hayabusa2 SRC with the Ryugu samples has landed in Woomera desert, Australia on December 6, 2020. After recovery of it, the gas extraction, collection into the bottles and measurements with the QMS were successfully operated using the GAEA. The analytical protocols are also presented here. The immediate gas extraction and handling technique described in this paper is likely applicable to future sample return missions.

\section{Abbreviations}

JAXA

Japan Aerospace Exploration Agency

ISAS

Institute of Space and Astronautical Science

WPA

Woomera Prohibited Area

QLF

Quick Look Facility

SRC

Sample Return Capsule

GAEA

GAs Extraction and Analyses system

HAWKS

HAyabusa2 gas collection With the Keyhole System 
QMS

Quadrupole mass spectrometer

CCG

Cold cathode gauge, MG:Miniature ion gauge

SEM

Secondary electron multiplier

\section{Declarations}

\section{Ethics approval and consent to participate}

Not applicable

\section{Consent for publication}

Not applicable

\section{Availability of data and materials}

The data used in this study are available from the corresponding author on reasonable request.

\section{Competing interests}

The authors declare that they have no competing interests.

\section{Funding}

The research is supported by the Hayabusa2 project (JAXA).

\section{Authors' contributions}

YNM summarized data and prepared the manuscript together with RO, ST and YT. RO and YNM made the design of the GAEA with contribution from YT, KS, ST, KY, SS and HS. YNM, RO and KS acquired preliminary experiments and calibration measurements. All authors contribute arrangement and improvement of the system, data reduction and interpretation. All authors read and approved the final manuscript.

\section{Acknowledgments}

We thank the members of the Astromaterials Science Research Group (ASRG) at JAXA and the Hayabusa2 curation team for assembling the entire working flow and cooperating in the rehearsal process. We would like to express our gratitude to Prof. Trevor Island (Australian National University) for witnessing and supervising the assembly and the actual analytical working flow as an international witness for the Hayabusa2 sample return capsule recovery at Woomera. We are grateful to Mr. S. Yamanouchi (Kyushu University) for his technical support to conduct fundamental experiments. 


\section{References}

1. Arakawa M, Saiki T, Wada K, Ogawa K, Kadono T, Shirai K, Sawada H, Ishibashi K, Honda R, Sakatani N, lijima Y, Okamoto C, Yano H, Takagi Y, Hayakawa M, Michel P, Jutzi M, Shimaki Y, Kimura S., Mimasu Y, Toda T, Imamura H, Nakazawa S, Hayakawa H, Sugita S, Morota T, Kameda S, Tatsumi E, Cho Y, Yoshioka K, Yokota Y, Matsuoka M, Yamada M, Kouyama T, Honda C, Tsuda Y, Watanabe S, Yoshikawa M, Tanaka S, Terui F, Kikuchi S, Yamaguchi T, Ogawa N, Ono G, Yoshikawa K, Takahashi T, Takei Y, Fujii A, Takeuchi H, Yamamoto Y, Okada T, Hirose C, Hosoda S, Mori O, Shimada T, Soldini S, Tsukizaki R, Iwata T, Ozaki M, Abe M, Namiki N, Kitazato K, Tachibana S, Ikeda H, Hirata N, Hirata N, Noguchi R, Miura A. (2020) An artificial impact on the asteroid162173 Ryugu formed a crater in the gravity-dominated regime. Science 368:67-71. https://doi. org/10.1126/science.aaz1701

2. Black DC (1972a) On the origins of trapped helium, neon and argon isotopic variations in meteorites - I. Gas-rich meteorites, lunar soil and breccia. Geochim Cosmochim Acta 36:347-375. https://doi.org/10.1016/0016-7037(72)90028-2

3. Black DC (1972b) On the origins of trapped helium, neon and argon isotopic variations in meteorites -I I. Carbonaceous meteorites. Geochim Cosmochim Acta 36:377-394.

https://doi.org/10.1016/0016-7037(72)90029-4

4. Busemann H, Meier MMM, Altmann F, Alwmark C, Bajt S, Beyersdorfer J, Böttger U, Crowther SA, Gilmour JD, Heitmann U, Hübers H-W, Maden C, Marone F, Pavlov SG, Schade U, Spring NH, Stampanoni M, Weber I (2015) New noble gas data and further examinations of dust from asteroid Itokawa. Paper presented at the 46th Lunar and Planetary Science Conference 46:\#2113

5. Hirashita, N., Urano, M. and Yoshida, H. (2013) Measurements of outgassing rages in a field of analysis (in Japanese with English abstract). J Vacuum Soc Japan 57:214-218. https://doi.org/10.3131/jvsj2.57.214

6. Jousten K (2008) Handbook of vacuum technology. Wiley-VCH, $1040 \mathrm{pp}$.

7. Kameda S, Yokota Y, Kouyama T, Tatsumi E, Ishida M, Morota T, Honda R, Sakatani N, Yamada M, Matsuoka M, Suzuki H, Cho Y, Hayakawa M, Honda C, Sawada H, Yoshioka K, Ogawa K Sugita S (2021) Improved method of hydrous mineral detection by latitudinal distribution of $0.7-\mu \mathrm{m}$ surface reflectance absorption on the asteroid Ryugu. Icarus 360:114348. https://doi:

10.1016/j.icarus.2021.114348

8. Kitazato K, Milliken RE, Iwata T, Abe M, Ohtake M, Matsuura S, Takagi Y, Nakamura T, Hiroi T, Matsuoka M, Riu L, Nakauchi Y, Tsumura K, Arai T, Senshu H, Hirata N, Barucci MA, Brunetto R, Pilorget C, Poulet F, Bibring J-P, Domingue DL, Vilas F, Takir D, Palomba E, Galiano A, Perna D, Osawa T. Komatsu M, Nakato A, Arai T, Takato N, Matsunaga T, Arakawa M, Saiki T, Wada K, Kadono T, Imamura H, Yano H, Shirai K, Hayakawa M, Okamoto C, Sawada H, Ogawa K, lijima Y, Sugita S, Honda R, Morota T, Kameda S, Tatsumi E, Cho Y, Yoshioka K, Yokota Y, Sakatani N, Yamada M, Kouyama T, Suzuki H, Honda C. Namiki N, Mizuno T, Matsumoto K, Noda H, Ishihara Y, Yamada R, Yamamoto K, Yoshida F, Abe S, Higuchi A, Yamamoto Y, Okada T, Shimaki Y, Noguchi R, Miura A, Hirata N, Tachibana S, Yabuta H, Ishiguro M, Ikeda H, Takeuchi H, Shimada T., Mori O, Hosoda S, 
Tsukizaki R, Soldini S, Ozaki M, Terui F, Ogawa N, Mimasu Y, Ono G, Yoshikawa K, Hirose C, Fujii A, Takahashi T, Kikuchi S, Takei Y, Yamaguchi T, Nakazawa S, Tanaka S, Yoshikawa M, Watanabe S, Tsuda Y (2021) Thermally altered subsurface material of asteroid (162173) Ryugu. Nature Astronomy 5:246-250. https://doi.org/10.1038/s41550-020-01271-2

9. Lieszkovsky L, Filippelli AR, Tilford CR (1990) Metrological characteristics of a group of quadrupole partial pressure analyzers. J Vacuum Sci Technol A 8:3838-3854. https://doi.org/10.1116/1.576458

10. Mahaffy PR, Trainer MG, Eigenbrode JL, Franz HB, Stern JC, Harpold DN, Conrad PG, Raaen E, Lyness E, the SAM Team (2011) Calibration of the quadrupole mass spectrometer of the Sample Analysis at Mars instrument suite. Paper presented at the 42nd Lunar and Planetary Science Conference:\#1556.

11. Malyshev OB, Middleman KJ (2008) In situ ultrahigh vacuum residual gas analyzer "calibration”. J Vacuum Sci Technol A 26:1474-1479. https://doi.org/10.1116/1.2990856

12. Morota T, Sugita S, Cho Y, Kanamaru M, Tatsumi E, Sakatani N, Honda R, Hirata N, Kikuchi H, Yamada M, Yokota Y, Kameda S, Matsuoka M, Sawada H, Honda C, Kouyama T, Ogawa K, Suzuki H, Yoshioka K, Hayakawa M, Hirata N, Hirabayashi M, Miyamoto H, Michikami T, Hiroi T, Hemmi R, Barnouin OS. Ernst CM, Kitazato K, Nakamura T, Riu L, Senshu H, Kobayashi H, Sasaki S, Komatsu G, Tanabe N, Fujii Y, Irie T, Suemitsu M, Takaki N, Sugimoto C, Yumoto K, Ishida M, Kato H, Moroi K, Domingue D, Michel P, Pilorget C, Iwata T, Abe M, Ohtake M, Nakauchi Y, Tsumura K, Yabuta H, Ishihara Y, Noguchi R, Matsumoto K, Miura A, Namik N, Tachibana S, Arakawa M, Ikeda H, Wada K, Mizuno T, Hirose C, Hosoda S, Mori O, Shimada T, Soldini S, Tsukizaki R, Yano H, Ozaki M, Takeuchi H, Yamamoto Y, Okada, T., Shimaki, Y., Shirai, K., lijima, Y., Noda, H., Kikuchi, S., Yamaguchi, T., Ogawa N, Ono G, Mimasu Y, Yoshikawa K, Takahashi T, Takei Y, Fujii A, Nakazawa S, Terui F, Tanaka S, Yoshikawa M, Saiki T, Watanabe S, Tsuda Y (2020) Sample collection from asteroid (162173) Ryugu by Hayabusa2: Implications for surface evolution. Science 368:654-659. https://doi.org/10.1126/science.aaz6306

13. Nagao K, Okazaki R, Nakamura T, Miura YN, Osawa T, Bajo K, Matsuda S, Ebihara M, Ireland TR, Kitajima F, Naraoka H, Noguchi T, Tsuchiyama A, Yurimoto H, Zolensky ME, Uesugi M, Shirai K, Abe M, Yada T, Ishibashi Y, Fujimura A, Mukai T, Ueno M, Okada T, Yoshikawa M, Kawaguchi J (2011) Irradiation history of Itokawa regolith material deduced from noble gases in the Hayabusa samples. Science 333:1128-1131. https://doi.org/10.1126/science.1207785

14. Okada T, Fukuhara T, Tanaka S, Taguchi M, Arai T, Senshu H, Sakatani N, Shimaki Y, Demura H, Ogawa Y, Kitazato K, Suko K, Sekiguchi T, Kouyama T, Takita J, Matsunaga T, Imamura T, Wada T, Hasegawa S, Helbert J, Müller TG, Hagermann A, Biele J, Grott M, Hamm M, Delbo M, Hirata N, Hirata N, Yamamoto Y, Terui F, Saiki T, Nakazawa S, Yoshikawa M, Watanabe S, Tsuda Y, Hayabusa2 TIR Team (2021) Thermophysical properties of C-type asteroid 162173 Ryugu revealed by the thermal infrared imager TIR on Hayabusa2. Trans JSASS Aerospace Tech Japan 19:654-659. https://doi.org/10.2322/tastj.19.654

15. Okazaki R, Nagao K, Miura YN, Osawa T, Bajo K, Matsuda S, Nakamura T, Shirai K, Abe M, Yada T, Noguchi T, Ishibashi Y, Fujimura A, Mukai T, Ueno M, Okada T, Yoshikawa M, Kawaguchi J (2011) 
Noble gases recovered from the Hayabusa sample container. Paper presented at the 42nd Lunar and Planetary Science Conference:\#1653

16. Okazaki R, Sawada H, Yamanouchi S, Tachibana S, Miura YN, Sakamoto K, Takano Y, Abe M, Itoh S, Yamada K, Yabuta H, Okamoto C, Yano H, Noguchi T, Nakamura T, Nagao K, The Hayabusa2 SMP Team (2017) Hayabusa2 sample catcher and container: Metal-seal system for vacuum encapsulation of returned samples with volatiles and organic compounds recovered from C-type asteroid Ryugu. Space Sci Rev 208:107-124. https:// doi.org/10.1007/s11214-016-0289-5

17. Ozima M, Podosek FA (2002) Noble Gas Geochemistry. Cambridge University Press, 367 pp.

18. Pepin RO, Becker RH, Schlutter DJ (1999) Irradiation records in regolith materials. I: Isotopic compositions of solar-wind neon and argon in single lunar mineral grains. Geochim Cosmochim Acta 63:2145-2162. https://doi.org/10.1016/S0016-7037(99)00002-2

19. Pilorget C, Okada T, Hamm V, Brunetto R, Yada T, Loizeau D, Riu L, Usui T, Moussi-Soffys A, Hatakeda K, Nakato A, Yogata K, Abe M, Aléon-Toppani A, Carter J, Chaigneau M, Crane B, Gondet B, Kumagai K, Langevin Y, Lantz C, Le Pivert-Jolivet T, Lequertier G, Lourit L, Miyazaki A, Nishimura M, Poulet F, Arakawa M, Hirata N, Kitazato K, Nakazawa S, Namiki N, Saiki T, Sugita S, Tachibana S, Tanaka S, Yoshikawa M, Tsuda Y, Watanabe S, Bibring JP (2021) First compositional analysis of Ryugu samples by the MicrOmega hyperspectral microscope. Nature Astronomy. https://doi.org/ 10.1038/s41550-021-01549-z

20. Saiki T, Takei Y, Takahashi T, Kikuchi S, Sawada H, Hirose C, Terui F, Ogawa N, Mimasu Y, Ono G, Yoshikawa K, Takeuchi H, Fujii A, Masuda A, Yasuda S, Matsushima K, Yoshikawa M, Nakazawa S, Tsuda Y, Hayabusa2 Project Team (2021) Overview of Hayabusa2 asteroidal proximity operation planning and preliminary results. Trans JSASS Aerospace Tech Japan 19:52-60. https://doi.org/10.2322/tastj.19.52

21. Sawada H, Okazaki R, Tachibana S, Sakamoto K, Takano Y, Okamoto C, Yano H, Miura YN, Abe M, Hasegawa S, Noguchi T, the Hayabusa2 SMP Team (2017) Hayabusa2 sampler: Collection of asteroidal surface material. Space Sci Rev 208:81-106. https://doi.org/10.1007/s11214-017-0338-8

22. Sugita S, Honda R, Morota T, Kameda S, Sawada H, Tatsumi E, Yamada M, Honda C, Yokota Y, Kouyama T, Sakatani N, Ogawa K, Suzuki H, Okada T, Namiki N, Tanaka S, lijima Y, Yoshioka K, Hayakawa M, Cho Y, Matsuoka M, Hirata N, Hirata N, Miyamoto H, Domingue D, Hirabayashi M, Nakamura T, Hiroi T, Michikami T, Michel P, Ballouz RL, Barnouin OS, Ernst CM, Schröder SE, Kikuchi H, Hemmi R, Komatsu G, Fukuhara T, Taguchi M, Arai T, Senshu H, Demura H, Ogawa Y, Shimaki Y, Sekiguchi T, Müller TG, Hagermann A, Mizuno T, Noda H, Matsumoto K, Yamada R, Ishihara Y, Ikeda H, Araki H, Yamamoto K, Abe S, Yoshida F, Higuchi A, Sasaki S, Oshigami S, Tsuruta S, Asari K, Tazawa S, Shizugami M, Kimura J, Otsubo T, Yabuta H, Hasegawa S, Ishiguro M, Tachibana S, Palmer E, Gaskell R, Le Corre L, Jaumann R, Otto K, Schmitz N, Abell P, A, Barucci MA, Zolensky ME, Vilas F, Thuillet F, Sugimoto C, Takaki N, Suzuki Y, Kamiyoshihara H, Okada M, Nagata K, Fujimoto M, Yoshikawa M, Yamamoto Y, Shirai K, Noguchi R, Ogawa N, Terui F, Kikuchi S, Yamaguchi T, Oki Y, Takao Y, Takeuchi H, Ono G, Mimasu Y, Yoshikawa K, Takahashi T, Takei Y, Fujii A, Hirose C, Nakazawa S, Hosoda S, Mori O, Shimada T, Soldini S, Iwata T, Abe M, Yano H, Tsukizaki R, Ozaki M, 
Nishiyama K, Saiki T, Watanabe S, Tsuda Y (2019) The geomorphology, color, and thermal properties of Ryugu: Implications for parent-body processes. Science

364:eaaw0422. https://doi.org/10.1126/science.aaw0422

23. Summers LR (1969) Empirical observations on the sensitivity of hot cathode ionization type vacuum gages. NASA Technical Note TND-5285, National Aeronautics and Space Administration, Washington, DC, June 1969

24. Sun W, Wu C, Cheng Y, Dong M, Guo M, Li Y, Ren Z, Jia W, Liu G, Wei N (2020) Study on calibrating the quadrupole mass spectrometers with gas mixture. Measurement 164:108099. https://doi.org/10.1016/j.measurement.2020.108099

25. Tachibana S, Abe M, Arakawa M, Fujimoto M, lijima $Y$, Ishiguro M, Kitazato K, Kobayashi N, Namiki N, Okada T, Okazaki R, Sawada H, Sugita S, Takano Y, Tanaka S, Watanabe S, Yoshikawa M, Kuninaka $\mathrm{H}$, the Hayabusa2 project team (2014) Hayabusa2: Scientific importance of samples returned from C-type near-Earth asteroid (162173) $1999 \mathrm{JU}_{3}$. Geochem J 48:571-587. https://doi.org/10.2343/geochemj.2.0350

26. Tachibana S., Sawada H, Okazaki R, Takano Y, Sakamoto K, Miura YN, Okamoto C, Yano H, Yamanouchi S, Michel P, Zhang Y, Schwartz S, Thuillet F, Yurimoto H, Nakamura T, Noguchi T, Yabuta H, Naraoka H, Tsuchiyama A, Imae N, Kurosawa K, Nakamura AM, Ogawa K, Sugita S, Morota T, Honda R, Kameda S, Tatsumi E, Cho Y, Yoshioka K, Yokota Y, Hayakawa M, Matsuoka M, Sakatani N, Yamada M, Kouyama T, Suzuki H, Honda C, Yoshimitsu T, Kubota T, Demura H, Yada T, Nishimura M, Yogata K, Nakato A, Yoshitake M, Suzuki Al, Furuya S, Hatakeda K, Miyazaki A, Kumagai K, Okada T, Abe M, Usui T, Ireland TR, Fujimoto M, Yamada T, Arakawa M, Connolly Jr. HC, Fujii A, Hasegawa S, Hirata N, Hirata N, Hirose C., Hosoda S, lijima Y, Ikeda H, Ishiguro M, Ishihara Y, Iwata T, Kikuchi S, Kitazato K, Lauretta DS, Libourel G, Marty B, Matsumoto K, Michikami T, Mimasu Y, Miura A, Mori O, Nakamura-Messenger K, Namiki N, Nguyen A N, Nittler L R, Noda H, Noguchi R, Ogawa N, Ono G, Ozaki M, Senshu H, Shimada T, Shimaki T, Shirai K, Soldini S, Takahashi T, Takei Y, Takeuchi H, Tsukizaki R, Wada K, Yamamoto Y, Yoshikawa K, Yumoto K, Zolensky ME, Nakazawa S, Terui F, Tanaka S, Saiki T, Yoshikawa M, Watanabe S, Tsuda Y (2021a) Pebbles and sans on asteroid (162173) Ryugu: On-site observation and returned particles from two landing sites. Submitted to Science (in Revision).

27. Tachibana S, Sawada H, Okazaki R, Takano Y, Sakamoto K, Miura YN, Yano H, Ireland TR, Nishimura M, Furuya S, Yamanouchi S, Maruyama T, Yada T, Nakato A, Yogata K, Kumagai K, Hatakeda K, Miyazaki A, Abe M, Usui T, Fujimoto M, Nakazawa S., Tsuda Y (2021b) Hayabusa2 reentry capsule retrieval and sample container opening operations. Paper presented at the 52nd Lunar and Planetary Science Conference:\#1289

28. Tatsumi E, Sugimoto C, Riu L, Sugita S, Nakamura T, Hiroi T, Morota T, Popescu M, Michikami M, Kitazato K, Matsuoka M, Kameda S, Honda R, Yamada M, Sakatani N, Kouyama T, Yokota Y, Honda C, Suzuki H, Cho Y, Ogawa K, Hayakawa M, Sawada H, Yoshioka K, Pilorget C, Ishida M, Domingue D, Hirata N, Sasaki S, de León J, Barucci MA, Michel P, Suemitsu M, Saiki T, Tanaka S, Terui F, Nakazawa S, Kikuchi S, Yamaguchi T, Ogawa N, Ono G, Mimasu Y, Yoshikawa K, Takahashi T, Takei 
Y, Fujii A, Yamamoto Y, Okada T, Hirose C, Hosoda S, Mori O, Shimada T, Soldini S, Tsukizaki R, Mizuno T, Iwata T, Yano H, Ozaki M, Abe M, Ohtake M, Namiki N, Tachibana S, Arakawa M, Ikeda H, Ishiguro M, Wada K, Yabuta H, Takeuchi H, Shimaki Y, Shirai K, Hirata N, lijima Y, Tsuda Y, Watanabe S, Yoshikawa M (2021) Collisional history of Ryugu's parent body from bright surface boulders. Nature Astronomy 5:39-45. https://doi.org/10.1038/s41550-020-1179-z

29. Tsuda Y, Saiki T, Terui F, Nakazawa S, Yoshikawa M, Watanabe S, Hayabusa2 Project Team (2020) Initial achievements of Hayabusa2 in asteroid proximity phase. Trans Japan Soc Aero Space Sci 63:115-123. https://doi.org/10.2322/tjsass.63.115

30. Watanabe S, Tsuda Y, Yoshikawa M, Tanaka S, Saiki T, Nakazawa S (2017) Hayabusa2 mission overview. Space Sci Rev 208:3-16. https://doi.org/10.1007/s11214-017-0377-1

31. Wieler R (2016) Do lunar and meteoritic archives record temporal variations in the composition of solar wind noble gases and nitrogen? A reassessment in the light of Genesis data. Chemie der Erde 76:462-480. https://doi.org/10.1016/j.chemer.2016.06.001

32. Wilfert S (2003) The contamination effect of cold cathode gauges. J Vacuum Soc Japan 46:31-37. https://doi.org/10.3131/jvsj.46.31

33. Yada T, Abe M, Okada T, Nakato A, Yogata K, Miyazaki A, Hatakeda K, Kumagai K, Nishimura M, Hitomi Y, Soejima H, Yoshitake M, Iwamae A, Furuya S, Uesugi M, Karouji Y, Usui T, Hayashi T, Yamamoto D, Fukai R, Sugita S, Cho Y, Yumoto K, Yabe Y, Bibring JP, Pilorget C, Hamm V, Brunetto R, Riu L, Lourit L, Loizeau D, Lequertier G, Moussi-Soffys A, Tachibana S, Sawada H, Okazaki R, Takano Y, Sakamoto K, Miura YN, Yano H, Ireland TR, Yamada T, Fujimoto M, Kitazato K, Namiki N, Arakawa M, Hirata N, Yurimoto H, Nakamura T, Noguchi T, Yabuta H, Naraoka H, Ito M, Nakamura E, Uesugi K, Kobayashi K, Michikami T, Kikuchi H, Hirata N, Ishihara Y, Matsumoto K, Noda H, Noguchi R, Shimaki Y, Shirai K, Ogawa K, Wada K, Senshu H, Yamamoto Y, Morota T, Honda R, Honda C, Yokota Y, Matsuoka M, Sakatani N, Tatsumi E, Miura A, Yamada M, Fujii A, Hirose C, Hosoda S, Ikeda H, Iwata T, Kikuchi S, Mimasu Y, Mori O, Ogawa N, Ono G, Shimada T, Soldini S, Takahashi T, Takei Y, Takeuchi H, Tsukizaki R, Yoshikawa K, Terui F, Nakazawa S, Tanaka S, Saiki T, Yoshikawa M, Watanabe S, Tsuda,Y (2021) Preliminary analysis of the Hayabusa2 samples returned from C-type asteroid Ryugu. Nature Astronomy. https://doi.org/10.1038/s41550-021-01550-6

34. Yamada T, Kawahara K, Itou T, Nakazawa S (2021) Return and recovery operation of the Hayabusa2 Sample Return Capsule. Trans JSASS Aerospace Tech Japan 19:514-522. https://doi.org/10.2322/tastj.19.514

35. Yoshida H, Arai K (2018) Quantitative measurements of various gases in high and ultrahigh vacuum. J Vacuum Sci Tech A 36:4031604. https://doi.org/ 10.1116/1.5018210

36. Yoshida H, Arai K, Kobata T (2014) In-situ calibration method for ionization gauges and quadrupole mass spectrometers by combining the standard conductance element and the conductance modulation method (SCEeCM method). Vacuum 101: 433-439. https://doi.org10.1016/j.vacuum.2013.05.015 


\section{Tables}

Tables are available in the Supplemental Files section.

\section{Figures}

\section{Figure 1}

Schematic draw of the Hayabusa2 sample container mounted on the ICF356 flange. The flange is equipped with a liner motion feed-through, a view port and a metal valve (VACGEN ZCR20). It is connected to the GAEA via the ICF34 port of the valve.

\section{Figure 2}

Gas sampling interface and needle for the HAyabusa2 gas collection With the Keyhole System (HAWKS). a cross section of the Hayabusa2 sample container (after Okazaki et al., 2017), b photograph for the gassampling interface of a dummy container, $\mathbf{c}$ dimension for the gas-sampling interface at the bottom of the sample container, where the metal filter adopted here is Swagelok SS-4-VCR-2-.5M (made of SS-316 and the defined pore size is $0.5-2 \mu \mathrm{m}$ ), and $\mathbf{d}$ schematic draw of the needle that we used for the actual operation at Woomera. The numbers are the sizes given in millimeters.

\section{Figure 3}

Configuration and result for the preliminary experiment about vacuum test using two types of pipes with different wall thicknesses. The test pipe-A has a thin thickness of $1.65 \mathrm{~mm}$, and the test pipe-B has a thick thickness of $14 \mathrm{~mm}$. The inner diameters are 6.2 and $6.0 \mathrm{~mm}$, respectively. a configuration of the experimental system, $\mathbf{b}$ photograph of the experimental system, and $\mathbf{c}$ result of the vacuum pressures measured with the gauges $\langle\mathrm{U}\rangle$ (upstream side) and $\langle\mathrm{D}\rangle$ (downstream side), as shown in figure 3a, against evacuating time. Two pipes show similar vacuum pressure transitions.

\section{Figure 4}

Schematic draw of the GAs Extraction and Analyses system (GAEA). Areas used for gas expansion are shown in blue, and those connected to evacuation systems are in grey. Each area between valves is named as labeled in italic. Abbreviation: QMS (W) - quadrupole mass spectrometer (Watmass), QMS (A) 
- quadrupole mass spectrometer (Anelva), SORB-AC - SORB-AC getter, CCG - cold cathode gauge, MG miniature ion gauge, PG - pirani gauge, TMP - turbo molecular pump, DSP - dry scroll pump.

\section{Figure 5}

External view of the GAEA. The numbers are the dimensions in millimeters.

\section{Figure 6}

Schematic diagram of power source distribution. The PLC performs valve operation and system status monitor.

\section{Figure 7}

Photographs of the GAEA set at the ISAS/JAXA (Sagamihara) and the QLF (Woomera).

\section{Figure 8}

Mass spectra for calibration gas measurements (calibration number of 10 (c), 11 (a) and 12) and a blank measurement. The mass spectra at $\sim 55 \mathrm{sec}$ after gas introduction (at top) and intensities of representative $m / z$ spectra with time (at bottom) are shown. The amounts of calibration gases (the terrestrial atmosphere) and the areas to which the calibration gases were introduced are indicated. Other calibration conditions are summarized in Table 3.

\section{Figure 9}

An outline of analytical protocols for extraction, storage and measurements of volatile gases in the Hayabusa2 sample container. RawGas means gases without any separation processes. DryGas means remaining gases after trapping gases in gas bottles of $\mathrm{CR} \# 1$ and \#2 at the liquid nitrogen temperature.

\section{Supplementary Files}

This is a list of supplementary files associated with this preprint. Click to download. 
- Additionalfile1Miura.pdf

- GraphicalAbstractMiurarev.jpeg

- TablesMiura.pdf 